\title{
Determinantes de la pobreza rural en México: Caso de 5 localidades
}

\section{Determinants of rural poverty in México: Case of 5 localities}

Plácido Salomón Álvarez López ${ }^{a}$

salomones@gmail.com
Elizabeth Trujillo Ubaldob

elizabeth.trubal@gmail.com

Karina Pérez Robles ${ }^{\mathrm{C}}$

karopr@hotmail.com

\section{Resumen}

Con el objetivo de abordar el esquema de la lucha contra la pobreza en México y la definición de estrategias para una política pública adecuada, se propone una metodología de análisis que identificó la probabilidad de riesgo en cinco localidades. Para esto, se identificó el grado de sensibilidad de las variables que explican la condición de pobreza de los jefes/jefas de hogar en 5 localidades rurales de México; con base en información recolectada a través de un censo, se formuló un modelo de regresión probabilístico, con el cual se estimaron tres tipos de indicadores: efectos marginales, elasticidades y razón de momios. Dentro de los principales resultados destaca que las variables como el ingreso, nivel educativo, hacinamiento y servicios en la vivienda presentan una relación inversa en la probabilidad, mientras que, alimentación, sexo, y hablar lengua indígena indican una relación directa en los factores que prevalecen sobre la probabilidad de que el jefe/jefa de hogar sea pobre. Concluyendo que los factores explicativos de naturaleza cualitativa y cuantitativa propios del jefe/jefa de hogar, en combinación a los relacionados con la vivienda y el hogar, permiten generar distribuciones de probabilidad consistentes con la argumentación teórica.

Palabras clave: Análisis del hogar, características de la vivienda, jefe/jefa del hogar, pobreza multidimensional.

\section{Abstract}

\footnotetext{
aDocente de la Universidad Autónoma de Guadalajara

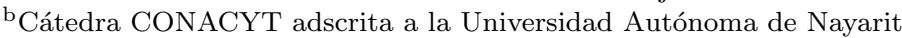

${ }^{\mathrm{c}}$ Cátedra CONACYT adscrita a la Universidad Autónoma de Nayarit
} 
With the objective of addressing the scheme of the fight against poverty in Mexico and the definition of strategies for an adequate public policy, an analysis methodology is proposed that identified the probability of risk in five localities affected by poverty. For this, the degree of sensitivity of the variables that explain the poverty condition of the heads of household in 5 rural locations in Mexico was identified; Based on information collected through a census, a probabilistic regression model was formulated, with which three types of indicators were estimated: marginal effects, elasticities and odds ratio. Among the main results, it is worth highlighting that variables such as income, educational level, overcrowding and housing services have an inverse relationship in probability, while food, sex, and speaking indigenous language indicate a direct relationship in the prevailing factors. about the probability that the head of household is poor. Concluding that the explanatory factors of qualitative and quantitative nature of the head of household, in combination with those related to housing and the home, allow generating probability distributions consistent with the theoretical argument.

Keywords: Analysis of the household, characteristics of the dwelling, head of the household, multidimensional poverty. 


\section{Introducción}

La pobreza en México presenta características diferenciadas según la región, etnia, zona rural o urbana, sin embargo, la población mexicana con mayores carencias sociales está compuesta en su mayoría de población rural. Es común, que las personas que nacen en hogares ubicados en zonas rurales se caractericen por bajos ingresos y bajos niveles de escolaridad o marginación, lo cual trae como consecuencias desventajas significativas que los limita para alcanzar su potencial humano, por tanto, en la actualidad y para el caso de México, no basta con el crecimiento económico para disminuir la pobreza de las zonas rurales (Damián y Boltvinik, 2003; Martínez et al., 2015; Ocampo, 2005). Es decir, el crecimiento económico de un país debe proyectar más, que el simple hecho de elevar sus indicadores macroeconómicos, priorizando en la relación recurrente con el bienestar de su población, siendo este el andamiaje que desvele los indicadores significativos entre el desempeño macroeconómico y una determinada condición individual (Melgar y Rossi, 2011). Para tal efecto, diferenciar entre los factores propios de cada población, permite determinar el impacto directo o residual en las variables que determinan la pobreza multidimensional del individuo, efectos derivados de la incapacidad de respuesta ante los riesgos que originan los ciclos económicos y la propia globalización (Damian, 2015; Destremau y Salama, 2002; Sen, 2001; Castillo y Arzate, 2013).

En cuestiones metodológicas y legales, se han logrado importantes acuerdos; respecto al tiempo y forma de recabar información, contribuyendo a la conceptualización, análisis y medición de la pobreza rural y urbana en México.

Respecto a las metodologías utilizadas para la mediciones de la pobreza, se destacan tres vertientes: 1) monetarias o líneas de pobreza (ingreso o gasto de los hogares); 2) no monetarias, se basan en cuantificar el rezago en indicadores de bienestar tales como la educación y salud; y 3) mixtas, se basan en combinar medidas monetarias y no monetarias, se destaca el método matricial, el método de medición integrado de la pobreza (MMIP) y el método del CONEVAL; que combinan los métodos de Línea de Pobreza y Necesidades Básicas Insatisfechas (Cortés y Hernández, 2004; Boltvinik, 2003; CONEVAL, 2010).

Además, el método del CONEVAL clasifica cuatro situaciones, las cuales se identifican en: pobres multidimensionales, vulnerables por carencias sociales, vulnerables por ingresos y no pobre multidimensional ni vulnerable; a su vez, en el cuadrante de pobreza multidimensional se divide en extrema y moderada (DOF, 2010). Bajo estos enfoques, se define la pobreza de la siguiente manera:

"Una persona se encuentra en situación de pobreza multidimensional cuando no tiene garantizado el ejercicio de al menos uno de sus derechos para el desarrollo social, y sus ingresos son insuficientes para adquirir los bienes y servicios que requiere para satisfacer sus necesidades" (CONEVAL, 2010: 38)

Comunicaciones en Estadística, diciembre 2019, Vol. 12, No. 2 
El concepto de pobreza empleado por el CONEVAL es el que en mayor medida se ajusta a las necesidades de esta investigación, por lo cual será empleado como eje central de la metodología aquí plasmada.

$\mathrm{Al}$ analizar los factores que integran la pobreza multidimensional, se puede llegar a disentir entre tantas dimensiones, como perspectivas puedan existir, el conceso reside en identificar los factores que la mantienen activa en diferentes circunstancias, tiempo y poblaciones disímiles entre si.

"al desarrollar una teoría de la pobreza, es tan importante entender la generación de nuevos estilos de vida, para establecer normas, servicios y costumbres a los que se espera puedan acceder los pobres, asi como entender que la generación y distribución de los recursos permite a la gente participar en esos estilos propios de vida" (Townsend citado en Damian, 2010: 28).

El análisis de la pobreza tiene modas y tendencias, y es así como entre las principales conceptualizaciones y factores que explican una determinada condición de pobreza se consideró la pobreza absoluta o primary poverty como aquella situación aplicada a familias cuyos ingresos fueron insuficientes para obtener el mínimo necesario para el mantenimiento -eficiencia psicológica- (Atkinson, 1987). Identificando también a la exclusión social como un proceso dinámico y determinante de la pobreza, aludiendo a diversas dimensiones: personales, laborales, económicas, sociales y culturales (Tezanos y Tezanos, 2004). Mediante el uso de los modelos logit y probit en investigaciones sociales, se encuentran resultados sobre aspectos como, que el acceso a activos públicos beneficia al desarrollo de activos privados de los jefes/jefas de hogar, integrando con esto, los principales factores de pobreza multidimensional en Perú, concluyendo que existe evidencia estadística para argumentar que dichos factores disminuyen la probabilidad de ser pobre (PucutayVázquez, 2002). Así mismo, la pobreza en que viven millones de personas, en parte se encuentra determinada por la exclusión, vulnerabilidad, discriminación y marginalidad, es así como la pobreza puede interpretarse mediante un análisis multidimensional, en el cual se involucren las carencias de capacidades fundamentales como criterio inherente, esto implica poner atención en las carencias socioeconómicas y circunstancias geográficas que las limitan (Sen, 1992; Townsend, 1993). De acuerdo con CONEVAL (2010) la medición de la pobreza se enmarca en un contexto multidimensional, en el cual se incorporan características como: 1) Ingreso, 2) Rezago educativo, 3) Acceso a servicios de salud, 4) Acceso a la seguridad social, 5) Calidad y espacios de la vivienda, 6) Acceso a los servicios básicos en la vivienda, 7) Acceso a la alimentación y 8) Grado de cohesión social.

Lo anterior en un intento por focalizar acciones de política social, puesto que, de acuerdo con Fernández - Vega (2013), el gasto que el gobierno (federal, estatal y municipal) destinó para la ejecución de programas de combate a la pobreza, de 1970 a 2012, incrementó $96.1 \%$ y el número de pobres continuaba en constante aumento (Ver Tabla 1). En 1988, el gobierno federal emprende el primer programa 
de lucha contra la pobreza en México, denominado Programa Nacional de Solidaridad Económica (Pronasol), posteriormente llamado Progresa, Oportunidades y Prospera.

Asimismo, en 2004, se promulga la Ley General de Desarrollo Social (LGDS), en la cual se dispuso la creación del Consejo Nacional de Evaluación de la Política de Desarrollo Social (CONEVAL), quien en 2008 y con apoyo del Instituto Nacional de Estadística y Geografía (INEGI), incorporaron en la Encuesta de Ingreso y Gasto de los Hogares (ENIGH) el Módulo de Condiciones Socioeconómicas (MCS), siendo así, uno de los primeros países del mundo en contar con una medición oficial multidimensional de la pobreza.

Tabla 1: Tasa de crecimiento promedio de la pobreza en México 1970-2018

\begin{tabular}{ccc}
\hline Año & Millones de pobres en México & TCM \\
\hline 1970 & $31,450,000$ & $0 \%$ \\
1980 & $38,675,000$ & $2.09 \%$ \\
1990 & $40,232,000$ & $0.40 \%$ \\
2000 & $48,000,650$ & $1.78 \%$ \\
2008 & $49,500,000$ & $0.39 \%$ \\
2010 & $52,000,000$ & $2.49 \%$ \\
2012 & $54,000,000$ & $1.90 \%$ \\
2014 & $55,000,000$ & $0.92 \%$ \\
2016 & $53,400,000$ & $-1.47 \%$ \\
2018 & $52,400,000$ & $-0.94 \%$
\end{tabular}

Fuente: Elaboración propia con datos del Centro de Análisis Multidisciplinario (CAM) y CONEVAL.

Los datos de pobreza en México han mostrado un panorama mixto, entre 2008 y 2018, la pobreza aumentó en 2.9 millones de personas al pasar de 49.5 a 52.4 , al mismo tiempo 3 millones de personas dejaron de estar en situación de pobreza extrema; en 2008 había 12.3 millones y en 2018, 9.3 millones. La población pobre presenta características diferenciadas según la condición étnica de las personas, la región en la que vivan y otras más. Por un lado, en las zonas rurales, la pobreza se encuentra en comunidades pequeñas y dispersas; por otro lado, el porcentaje de pobreza en las zonas urbanas si bien es menor, el número de personas en esa condición es mayor (CONEVAL, 2018).

La presente investigación aborda el análisis de los factores determinantes de la pobreza para 5 localidades rurales en México. Los estudios empíricos sobre este tema para México son escasos, especialmente con un abordaje cuantitativo y diferenciado por zonas, con excepción de los estudios de Ponce (2012) y Cortés (1997), quienes utilizaron los datos de la Encuesta Nacional de Ingreso Gasto de los Hogares (ENIGH) 2010 y la Encuesta de Ingresos y Gastos de 1992, respectivamente y sometieron a pruebas simultáneas las diversas explicaciones que identificaron en la literatura sobre los determinantes que explican la condición de pobreza de un hogar. 
En el presente trabajo, se analiza la situación de pobreza en sus múltiples dimensiones que reflejan las localidades objeto del estudio, y el peso o sensibilidad de respuesta a las diferentes variables que caracterizan al jefe/jefa del hogar, en combinación con las condiciones propias del hogar y de la vivienda, siendo el objetivo general, identificar el grado de sensibilidad o capacidad de respuesta de las variables que explican la condición de pobreza de los jefes/jefas de hogar de cinco localidades rurales de México. Como objetivo específico se planteó, determinar sí el modelo de regresión probabilístico logit, es robusto para ajustar modelos de pobreza y coadyuvar a la definición de estrategias para una política pública dirigida.

\section{Enfoque metodológico}

\subsection{Fuente y datos de información}

La presente investigación se enmarca en una investigación correlacional, en la modalidad de estudio de caso, en el ámbito estatal llevado a cabo en el año 2011. Para la selección de los casos de estudio se identificaron los estados que registraron un mayor porcentaje de población con ingreso inferior a la Línea de Bienestar (LB); Oaxaca fue el estado que registró el mayor porcentaje (69\%), seguido por Guanajuato (54\%), Estado de México (48\%) y Querétaro (46\%). En cuanto a la población con ingreso inferior a la Línea de Bienestar Mínimo (LBM); Oaxaca se mantuvo con el mayor porcentaje (37\%), seguido por Guanajuato (17\%), Querétaro (16\%) y Estado de México (14\%) (CONEVAL, 2011). De estos 5 estados, se seleccionaron las localidades bajo dos criterios: 1) zonas prioritarias para la Secretaria de Desarrollo Social (SEDESOL) y 2) municipios sin intervención previa a 2011 por la Iniciativa de Apoyo Integral para la Superación de la Pobreza. Una vez identificadas las localidades con estas características, se seleccionaron las cinco con mayor heterogeneidad de acuerdo con las siguientes variables: grado de marginación, tamaño de la población, ubicación geográfica y actividades económicas que se desarrollan (Sagarnaga y Salas, 2011). Se levantó un censo en las localidades seleccionadas, San José Pie del Cerro, Oaxaca; Sangre de Cristo, Guanajuato; La Higuera y El Paraíso, Querétaro; y Pachuquilla, Estado de México (ver Figura 1).

La población objeto de estudio se integró en 311 hogares; por medio de entrevistas cara a cara a las personas jefe/jefa de hogar, en el periodo de mayo a septiembre de 2011, se lograron 266 entrevistas completas (85.5\% de respuesta). El cuestionario se dividió en cuatro módulos: vivienda, hogar, personas mayores de 12 años y personas menores de 12 años. Se obtuvo información de los siguientes temas: Identificación del hogar, características sociodemográficas, alimentación, percepción de la salud, disponibilidad de agua, disposición de excretas, drenaje, educación, vivienda, ingreso, gasto, medio ambiente, servicios básicos y distancias a los principales puntos de interés.

Las carencias sociales de las localidades y de la población bajo estudio se sintetizan

Comunicaciones en Estadística, diciembre 2019, Vol. 12, No. 2 
Figura 1: Localidades de estudio.

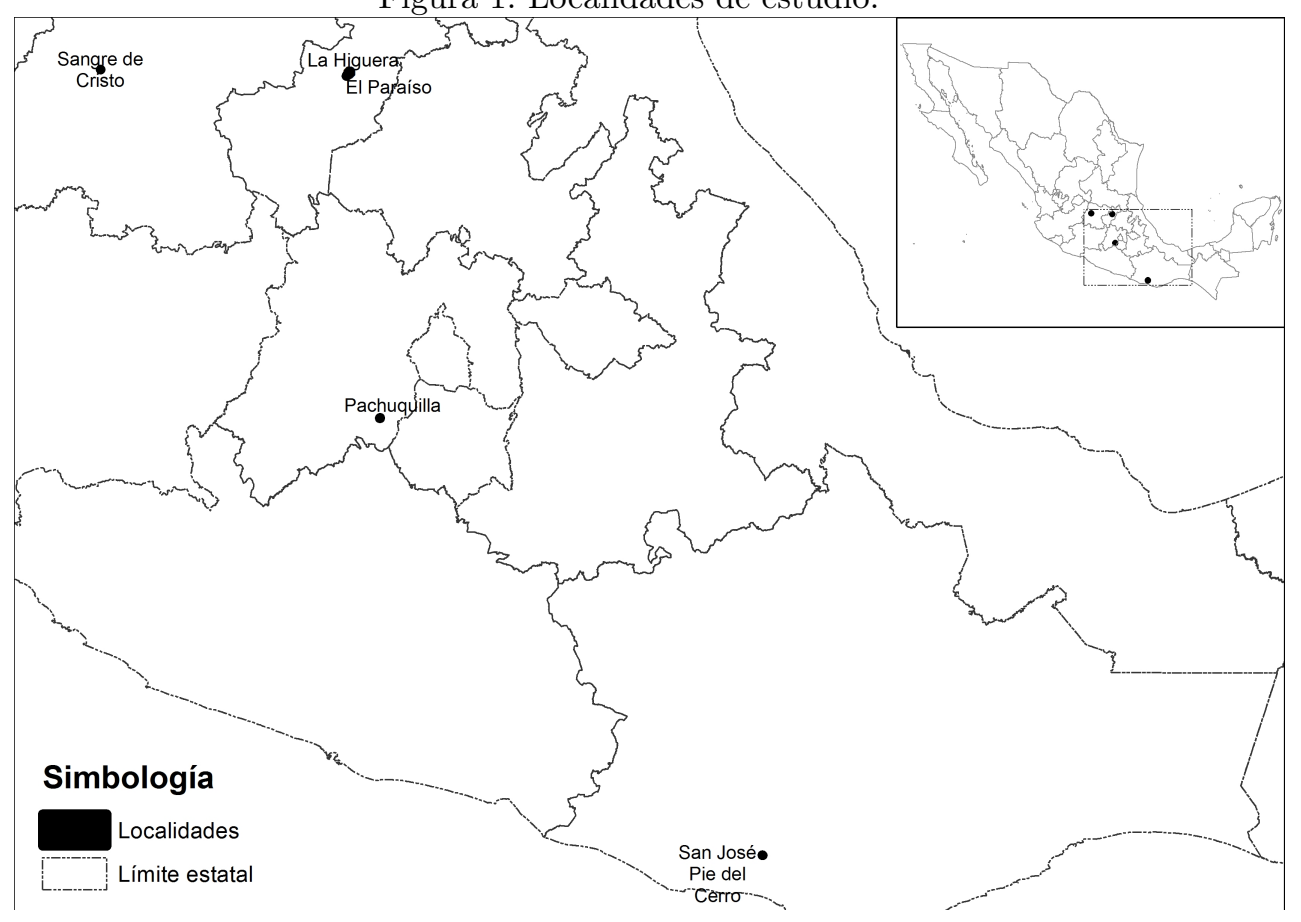

Fuente: Elaboración propia. 
Tabla 2: Tipo de carencia por localidad

\begin{tabular}{lcccccc}
\hline Localidad & Alimentación & $\begin{array}{c}\text { Rezago } \\
\text { educativo }\end{array}$ & $\begin{array}{c}\text { Servicios } \\
\text { de salud }\end{array}$ & $\begin{array}{c}\text { Seguridad } \\
\text { social }\end{array}$ & $\begin{array}{c}\text { Calidad y } \\
\text { espacios } \\
\text { vivienda } \\
\%\end{array}$ & $\begin{array}{c}\text { Servicios } \\
\text { básicos de } \\
\text { la vivienda } \\
\%\end{array}$ \\
\hline El Paraíso & 17 & $\%$ & $\%$ & $\%$ & $\%$ & $\%$ \\
La Higuera & 27 & 39 & 0 & 96 & 81 & 100 \\
Pachuquilla & 32 & 36 & 9 & 97 & 57 & 95 \\
San José & 64 & 52 & 18 & 99 & 72 & 98 \\
Sangre de & 17 & 26 & 12 & 90 & 36 & 100 \\
Cristo & & & & & & \\
\hline \multicolumn{1}{c}{ Total } & $\mathbf{3 3}$ & $\mathbf{3 6}$ & $\mathbf{1 1}$ & $\mathbf{9 5}$ & $\mathbf{5 7}$ & $\mathbf{9 6}$ \\
\hline
\end{tabular}

Fuente: elaboración a partir del levantamiento de información.

en la Tabla 2. Las carencias más importantes son: servicios básicos de la vivienda, calidad y espacios de la vivienda y rezago educativo. Un hallazgo importante es que el acceso a servicios de salud no resultó como una carencia significativa en ninguna de las localidades, debido a la percepción relacionada con la cobertura del Seguro Popular.

Con esta información se procedió a evaluar la pobreza multidimensional de los habitantes de las localidades estudiadas, siendo que el $32 \%$ de la población objeto de estudio, se encontró en pobreza extrema y $46 \%$ en pobreza moderada, lo cual justificó su elección para el desarrollo de la metodología propuesta.

\subsection{Análisis de los datos}

Para identificar la pobreza multidimensional de la población objeto de estudio, se determinaron dos cantidades mínimas de recursos monetarios requeridas para satisfacer las necesidades básicas de las personas, representadas por dos canastas básicas: una alimentaria y otra no alimentaria. Estas cantidades monetarias definen la LBM y LB respectivamente. Para estimar el nivel de pobreza, se utilizó el ingreso mensual promedio corriente de los hogares (periodo mayo a octubre de 2011); a partir de éste, se calculó el ingreso mensual promedio corriente per cápita, dividiendo entre el número equivalente de miembros del hogar (DOF, 2010; 50). Las LB y LBM tomadas como referencia para determinar los niveles de pobreza fueron de 1,376.32y713.53 pesos, que son los valores promedio definidos por el CONEVAL para las zonas rurales del país durante el periodo mayo-septiembre de 2011.

El ingreso de los hogares de las localidades incluidas en el estudio fue medido con el fin de usarlo como indicador indirecto para medir el nivel de bienestar económico y determinar los niveles de pobreza de la población. Para cuantificar el ingreso de los hogares, se integró la suma de las percepciones de todos los miembros del 
hogar recibidas en el periodo mayo-octubre de 2011, tanto las monetarias como las no monetarias. De acuerdo con esta tipificación se procedió al procesamiento de los datos, para así adecuar la información obtenida en campo con el desarrollo de los modelos probabilísticos de regresión con repuestas cualitativas. Este tipo de modelos, se componen por una variable dependiente de orden cualitativa y variables independientes de naturaleza cuantitativa, cualitativa o una mezcla de ambas (Moscote y Rincón, 2012: 124). Existen diversos modelos de elección discreta y de naturaleza cualitativa como, por ejemplo: modelos lineales de probabilidad (MLP), modelo tobit, modelo probit y modelo logit. Los dos primero presentan algunas dificultades teóricas para aproximar modelos de variables dicotómicas, y los dos últimos han mostrado ser robustos en términos teóricos y estadísticos (Greene, 2003: 719). Para esto, se dice que:

"El uso de una función de distribución garantiza que el resultado de la estimación esté acotado entre 0 y 1, en principio las posibles alternativas son varias, siendo las más habituales la función de distribución logística, que ha dado lugar al modelo Logit, y la función de distribución de la normal tipificada, que ha dado lugar al modelo Probit" (Rodríguez y Cáceres, 2007: 453).

Según esta aproximación, entre las curvas de la distribución normal tipificada y de la distribución logística, los parámetros estimados por dichos modelos difieren en grado mínimamente entre sí. Por lo anterior, al mostrar ambos modelos diferencias significativas mínimas, se utilizó el modelo probabilístico logit, donde la variable exógena (pobreza de los jefes/jefas de hogar de las cinco localidades en estudio) tomó los siguientes valores:

$$
Y_{i}=\left\{\begin{array}{l}
0, \quad i \text {-ésima jefe } / \text { jefa es no pobre } \\
1, \quad i \text {-ésima jefe } / \text { jefa es pobre }
\end{array}\right.
$$

Por lo tanto, la restricción es $0 \leq E\left(Y_{i} \mid x_{i}\right) \leq 1$. Nótese que el modelo logit, está conformado por una función monótona creciente en forma de $S$ (función logística), y suele utilizarse con frecuencia en este tipo de estudios por las siguientes razones: desde el punto de vista econométrico, es una función flexible y fácil de utilizar, tiene interpretaciones relativamente sencillas y la evidencia empírica ha demostrado que este modelo es adecuado en la mayoría de los casos en los cuales la respuesta es binaria.

El modelo logit representa la probabilidad de que el jefe/jefa de hogar sea pobre $\left(Y_{i}=1\right)$, se utiliza la función de distribución logística, y el modelo toma la siguiente forma (Greene, 2003):

$$
E\left(Y_{i}\right)=\frac{e^{x_{i}^{\prime} \beta}}{1+e^{x_{i}^{\prime} \beta}} \forall-\infty<x_{i}^{\prime} \beta<\infty,
$$


donde, $x$ es el vector de variables independientes y $\beta$ el vector de parámetros. Se estimó la probabilidad en un modelo logit, mediante la función de verosimilitud representada de la siguiente forma (Pucutay-Vázquez, 2002: 35):

$$
\begin{gathered}
\ln L=\sum_{i=1}^{N} Y_{i}\left(x_{i}^{\prime} \beta\right)-\sum_{i=1}^{N} \ln \left(1-e^{x_{i}^{\prime} \beta}\right) \\
\left(\sum_{i=1}^{N} Y_{i} x_{i}^{\prime}\right) \beta-\sum_{i=1}^{N} \ln \left(1+e^{x_{i}^{\prime} \beta}\right)=1-F(X ; B),
\end{gathered}
$$

aquí, el vector de párametros $(\beta)$ refleja el aporte que $x$ (vector de variables explicativas) tiene como parte de la funcion de distribución acumulada. Se tiene la siguiente representación de la condición de que el jefe/jefa de hogar sea pobre:

$$
P_{i}=E\left(Y_{i}=1 \mid X_{i}\right)=\frac{1}{1+e^{-x_{i}^{\prime} \beta}}
$$

para fines prácticos:

$$
x_{i}^{\prime} \beta=z_{i}
$$

y para facilidad del análisis evaluamos $x_{i}^{\prime} \beta$ en (4), obteniendo la siguiente expresión

$$
P_{i}=\frac{1}{1+e^{-z_{i}}}=\frac{e^{z_{i}}}{1+e^{z_{i}}}
$$

entonces la probabilidad de que el jefe/jefa de hogar no sea pobre, está dado por:

$$
\left(1-P_{i}\right)=\frac{1}{1+e^{z_{i}}}
$$

\subsection{Efectos marginales, elasticidades y razón de probabilida- des (odds ratio)}

Dentro de la economía existen dos conceptos que dan cuenta del cambio en las variables endógenas y exógenas, los indicadores de mayor utilidad son: efectos marginales y elasticidades. A continuación, se presentan la derivación de cada uno: 
- Efectos marginales (EMg):

$$
E M g=\frac{\partial P_{i}}{\partial X_{i}}=\frac{\partial\left(\frac{e^{z_{i}}}{1+e^{z_{i}}}\right)}{\partial X_{i}}=\frac{\partial P_{i}}{\partial z_{i}} * \frac{\partial z_{i}}{\partial X_{i}}=\beta_{i} P_{i}\left(1-P_{i}\right)
$$

- Elasticidades $\left(\varepsilon_{i}\right)$ :

$$
\varepsilon_{i}=\frac{\partial P_{i}}{\partial X_{i}} * \frac{x_{i}}{P_{i}}=\beta_{i} P_{i}\left(1-P_{i}\right) \frac{x_{i}}{P_{i}}=\beta_{i} x_{i}\left(1-P_{i}\right)
$$

Otra expresión usada en los modelos logit, es de la razón de probabilidades, la cual se expresa de la siguiente forma:

$$
\frac{P_{i}}{1-P_{i}}=\frac{1-e^{z_{i}}}{1+e^{-z_{i}}}=e^{z_{i}}
$$

Donde, $P_{i} /\left(1-P_{i}\right)$ es la razón de probabilidades a favor de que el jefe/jefa de hogar sea pobre (la razón de la probabilidad de que el jefe/jefa de hogar sea pobre respecto de la probabilidad de que no lo sea). Ahora bien, si se toma el logaritmo natural de la ecuación (10), se obtiene como resultado (Gujarati, 2004: 562):

$$
L=\ln \left(\frac{P_{i}}{1-P_{i}}\right)=Z
$$

Donde, $L$ es el logaritmo de la razón de probabilidades, el cual es lineal en $X$ y lineal en los parámetros (desde el punto de vista de estimación).

El software estadístico utilizado fue The Statistical Analysis System (SAS) versión 9.0 para Windows utilizando el procedimiento Proc Logistics del módulo SAS/STAT.

\section{Resultados y discusión}

Con base en los resultados del estudio, se determinó que en dos de las cinco localidades analizadas (Pachuquilla y San José Pie del Cerro), $90 \%$ de los jefes/jefas de hogar presentaron ingresos inferiores a la LB.

Se ajustaron diversos modelos de elección binaria logit para la variable dependiente $Y_{i}$, esto permitió identificar las variables independientes que presentaron mayor grado de significancia (se aplicaron pruebas estadísticas individuales y conjuntas). Para la selección del modelo óptimo se utilizó el criterio de información de Akaike (AIC), de Schwarz (SC) y la prueba del cociente de verosimilitud (ver Tabla 3) (Allison, 1999, p. 143). La estimación de estos criterios consideró dos supuestos: 1) términos independientes y 2 ) variables adicionales. 
Tabla 3: Criterios de ajuste del modelo

\begin{tabular}{lcc}
\hline Criterio & $\begin{array}{c}\text { Sólo Términos } \\
\text { independientes }\end{array}$ & $\begin{array}{c}\text { Términos independientes Y } \\
\text { Variables Adicionales }\end{array}$ \\
\hline AIC & 330.135 & 222.796 \\
SC & 333.649 & 289.551 \\
2 LOG L & 328 & 184.796 \\
\hline
\end{tabular}

Fuente: Elaboración propia con datos recabados en campo.

Se realizaron diversas iteraciones hasta obtener el modelo óptimo y las pruebas de significancia individual de Wald apropiadas (ver Tablas 4 y 5). Se estimaron 19 parámetros, de los cuales, uno está asociado al intercepto y 18 a las variables con mayor significancia, confirmando la tendencia asociada a cada variable, las cuales muestran un comportamiento teórico consistente (signos esperados).

Se examinó la consistencia del modelo mediante la prueba de razón de verosimilitud (LR por sus siglas en inglés); se observó un valor del estadístico $\chi^{2}$ igual a 143.3392 $(p$-value $=0.0001)$, por lo tanto, se concluyó que no existe evidencia estadística a favor de la hipótesis nula (hipótesis nula: todos los parámetros son iguales) en favor de la alternativa (al menos un parámetro es diferente de cero), asimismo, se obtuvo el estadístico Wald igual a 63.4728 ( $p$-value $=0.001)$.

Se resalta el hecho de las variables: estado de salud, hacinamiento, agua en la vivienda y sanitario, las cuales no fueron significativas a $10 \%$, sin embargo, se consideraron en el modelo por la importancia teórica y el alto estadístico global que ajustaron (ver Tabla 5).

Para explicar el ajuste global del modelo se utilizó el estadístico pseudo de McFadden (43\%), (Bateman, et. al, 2002), el ajuste del modelo fue satisfactorio, se evaluó la presencia de multicolinealidad a partir de la tolerancia (TOL) y del factor de inflación de la varianza (VIF), sí $T O L<0,1$ y $V I F \geq 10$ entonces existe multicolinealidad, para este análisis se obtuvieron $T O L>0.1$ y $V I F<10$, por lo tanto, existe evidencia estadística de no multicolinealidad de acuerdo con (Kennedy, 1992; Hair et. al, 1995). Por otra parte, de las 18 variables consideradas en el modelo, dos son continuas (ingreso e índice de hacinamiento) y el resto son categóricas.

En la Tabla 5 se observan las estimaciones de los efectos marginales promedio, los cuales son interpretados de acuerdo con los valores que toma cada una de las variables. Por ejemplo, el efecto marginal del ingreso promedio, por una variación positiva de una unidad en el ingreso, la probabilidad de ser pobre disminuye 0.000018 unidades. Nótese que el ingreso es una variable continua y toma valores mayores en comparación al resto. Para el caso de género, el efecto marginal es mayor en valor absoluto, pero el incremento máximo en la probabilidad de no ser pobre es de 0.100458 , dado que, por su naturaleza es dicotómica $(0=$ mujer y $1=$ hombre). 
166Plácido Salomón Álvarez López, Elizabeth Trujillo Ubaldo \& Karina Pérez Robles

Tabla 4: Descripción de las variables utilizadas en em modelo

\begin{tabular}{|c|c|c|}
\hline Abreviatura & Variable & Descripción \\
\hline $\mathrm{Y}$ & Probabilidad & $\begin{array}{l}\text { Condición de pobreza del jefe o jefa de hogar (por } \\
\text { debajo de la línea de pobreza extrema) }\end{array}$ \\
\hline Ingre & Ingreso & Ingreso mensual monetario del jefe o jefa del hogar \\
\hline Gene & Sexo & Sexo del jefe o jefa del hogar \\
\hline Habla_lengua & Lengua & $\begin{array}{l}\text { indígena o dialecto } \mathrm{El} \text { jefe o jefa de hogar habla al- } \\
\text { guna lengua indígena o dialecto }\end{array}$ \\
\hline Gradoaprob & Grado & aprobado Años de escolaridad del jefe o jefa del hogar \\
\hline Edoconyugal & Estado & conyugal Situación conyugal del jefe o jefa del hogar \\
\hline Edosalud & Estado de salud & $\begin{array}{l}\text { Percepción sobre la condición de salud del jefe o jefa } \\
\text { del hogar }\end{array}$ \\
\hline Alim & Alimentación & $\begin{array}{l}\text { Percepción sobre la suficiencia de la alimentación en } \\
\text { el hogar }\end{array}$ \\
\hline Alim18 & $\begin{array}{l}\text { Alimentación de los } \\
\text { menores de } 18 \text { años }\end{array}$ & $\begin{array}{l}\text { Percepción sobre la suficiencia en la alimentación de } \\
\text { los menores de } 18 \text { años en el hogar }\end{array}$ \\
\hline Mism_gast & Mismo gasto & $\begin{array}{l}\text { En el hogar sólo una persona percibe un ingreso men- } \\
\text { sual }\end{array}$ \\
\hline Viv_tiene & La vivienda tiene & Servicios que se encuentran en la vivienda \\
\hline Apar & $\begin{array}{l}\text { Aparatos en la vivien- } \\
\text { da }\end{array}$ & Electrodomésticos que se encuentran en la vivienda \\
\hline Mat_techo & Material del techo & Material de construcción del techo de la vivienda \\
\hline Mat_piso & Material del piso & Material del que está hecho el piso \\
\hline Hacin & Hacinamiento & $\begin{array}{l}\text { Número de habitantes dividido entre el número de } \\
\text { cuartos }\end{array}$ \\
\hline Agua_viv & Agua en la vivienda & Tipo de subministro del agua en la vivienda \\
\hline Sanit & Sanitario & Tipo de sanitario con el que cuenta la vivienda \\
\hline Sanit_comp & Sanitario compartido & El sanitario lo comparte con otra vivienda \\
\hline Bas & Basura & Tipo de recolección de los desechos \\
\hline
\end{tabular}

Fuente: Elaboración propia.

Respecto a las elasticidades, se estimó la sensibilidad de la probabilidad de que el jefe/jefa hogar sea pobre respecto a cada una de las variables. Por ejemplo, por una variación promedio de uno por ciento en el ingreso, el porcentaje en que varía la probabilidad de que el jefe/jefa de hogar sea pobre disminuye aproximadamente $0.73 \%$. Para el fenómeno de estudio se observó que la probabilidad de que el jefe/jefa de hogar sea pobre con respecto a las variables de interés son inelásticas, excepto para la variable mismo gastos, que mostró ser elástica.

En cuanto a las características propias del jefe/jefa de hogar, la variable ingreso tiene una elasticidad promedio de -0.7259 , resultando ser una variable estadísticamente significativa para ayudar a disminuir la probabilidad de que los jefes/jefas de hogar de estas localidades sean pobres.

La interpretación de la razón de probabilidades depende del tipo de escala de 
Tabla 5: Resultados del modelo ajustado

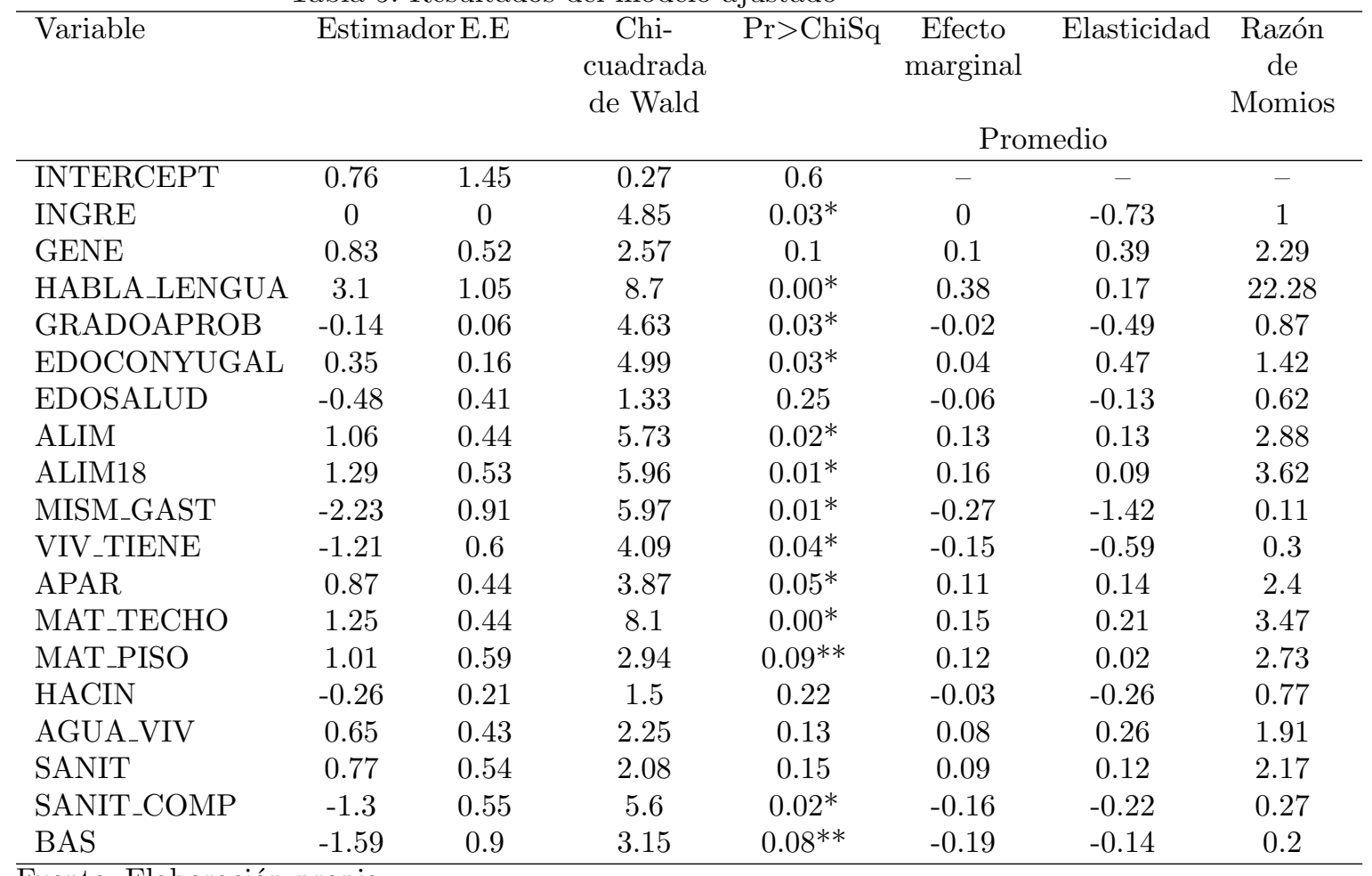

Fuente: Elaboración propia.

medición de las variables explicativas (Mandeville, 2008: 116). Para variables explicativas continuas, como por ejemplo el ingreso, la razón de probabilidad de que el jefe/jefa de hogar sea pobre es 0.9999 veces que la probabilidad de tener un ingreso bajo, manteniendo las otras variables explicativas constantes. Por otro lado, debido a que la razón de probabilidades es menor a 1 , se puede interpretar el reciproco $(1 / 0.9999=1.0001)$, de la siguiente forma, - si el jefe/jefa de hogar tiene un peso menos, entonces la probabilidad de ser pobre es 1.0001 veces mayor que si tuviera ese peso-, manteniéndose las demás variables constantes.

La variable grado aprobado, muestra que, a mayor grado aprobado disminuye la probabilidad de ser pobre con respecto a no serlo, el resultado de la razón de momios fue 1.144651, es decir, si el jefe/jefa de hogar tuviese un año menos de escolaridad, entonces la probabilidad de ser pobre aumentaría 1.144 veces más que si tuviera ese grado de escolaridad.

Para el caso del índice de hacinamiento, la razón de momios fue de 1.292 a razón de que a 1 habitante más por cuarto la probabilidad de que el jefe/jefa de hogar sea pobre aumenta 1.292 veces, es decir, si el promedio es de 3 personas por cuarto; si este se incrementa a 4 esta probabilidad aumenta. 
Para el caso de variables explicativas discretas, la interpretación es distinta. Por ejemplo: para el caso de género, si el jefe/jefa de hogar es hombre entonces la probabilidad de que este sea pobre es 2.28 veces mayor que si fuera mujer. Contrario a los resultados de otros estudios, se puede explicar que en estas comunidades se entrevistó a un mayor número de jefes/jefas de hogar hombres que cayeron en la línea de pobreza. En mucho de estos casos las mujeres percibían mayores ingresos provenientes de remesas o la presencia de un mayor número de miembros activos dentro del hogar.

Con base en los resultados, la variable "habla alguna lengua indígena" arrojó un coeficiente mayor, con respecto a las demás variables, por lo que se puede explicar que esta variable es la de mayor peso en la explicación de la pobreza, lo que indica que la razón de momios es de 22.3. Es decir, la razón de probabilidades de ser pobre contra no serlo es 22.3 veces más para el grupo de personas que hablan una lengua indígena comparada con la del grupo de personas que no hablan una lengua indígena sobre la esclusion y marginalidad que acentúa la pobreza (Arriagada, 2005; Córdova, 2011; Tezanos y Tezanos, 2004). En estudios reciente se ha demostrado que el hablar lengua indígena, pertenecer a cierta región del país y ser mujer eleva las probabilidades de ser pobre, por el contrario, los resultados de esta investigación demuestran que la probabilidad de ser pobre disminuye si la jefatura del hogar se encuentra a cargo de una mujer (Cortés, 1997). Para el caso de la variable estado de salud del jefe/jefa de hogar: la razón de momios es de 0.62 . Es decir, la razón de probabilidades de ser pobre contra no serlo, de un jefe/jefa de hogar que se encuentra en condiciones de enfermedad es aproximadamente 0.62 veces mayor que la del grupo con jefes/jefas de hogar que se perciben en condiciones buenas de salud física. En cuanto a la alimentación tanto en mayores como en menores de edad arrojó un signo positivo y una razón de momios de 2.87 y 3.61 respectivamente, es decir, si la percepción del jefe/jefa del hogar sobre la alimentación de los menores de 18 años es deficiente entonces la probabilidad de ser pobre es 3.61 veces mayor que si esta percepción fuera de una buena alimentación. Si la vivienda no cuenta con agua entubada ya sea dentro o fuera de la vivienda, la probabilidad de que el jefe/jefa del hogar sea pobre es de 1.91 veces mayor que si la vivienda contara con este servicio.

En el caso del material de la vivienda, resultó que, en hogares con viviendas de material de techo como la paja, aumenta la probabilidad de que el jefe/jefa del hogar sea pobre 3.47 veces más que los hogares con viviendas con material de techo como el concreto. Para el caso del material de piso, el jefe/jefa del hogar tiene una probabilidad de 2.73 veces más, comparado con los hogares con material de piso como el cemento.

En contra parte, para el caso de las cinco localidades rurales objeto de estudio de esta investigación, se encontró que el bienestar si tiene una alta relación con el género de la jefatura del hogar, el nivel de escolaridad y la composición del hogar (Ponce, 2012: 44). 


\section{Conclusiones}

En las últimas décadas se han registrado progresos importantes en el desarrollo social, sin embargo, los retos en materia de superación de la pobreza aún son grandes y persistentes.

La comparación de las 5 localidades estudiadas permitió identificar variables diferenciales que precisan la condición de pobreza de los jefes/jefas de hogar en comunidades rurales, estas variables son independientes y ejercen un peso de influencia sobre la probabilidad de caer en la pobreza. Los resultados contribuyen al sustento empírico y aportan evidencia estadística sobre la influencia de: el ingreso, hablar lengua indígena, suficiencia en su alimentación, educación, percepción del estado de salud, género; estas características se encuentran presentes en el jefe/jefa de hogar, mientras que, las propias de la vivienda, si cuenta con: agua, sanitario, material del que se encuentra hecha la vivienda y recolección de basura. Y, por último, variables referentes al hogar: aportaciones económicas al hogar o número de personas que habitan en la vivienda.

En consecuencia, los factores explicativos de naturaleza cualitativa y cuantitativa propios del jefe/jefa de hogar, en combinación a los relacionados con la vivienda y el hogar; permiten generar modelos ajustados a la probabilidad de ser pobre del jefe/jefa de hogar, siguiendo el enfoque de los modelos de regresión cualitativos.

El atomizar el concepto de pobreza, en variables o factores que se relacionen con las necesidades humanas, vinculando estas a entornos sociales y al contexto económico, así como, a los aspectos culturales, dará cuenta de estrategias adecuadas y dirigidas a corregir el entramado que constituye a la pobreza, de acuerdo con el peso y tendencia de cada una de estas variables como condicionantes de vulnerabilidad social, logrando así, mitigar el riesgo y prevalencia de la pobreza del individuo de las zonas rurales.

Recibido: Marzo de 2019 Aceptado: Enero de 2020

\section{Referencias}

Allison, P. (1999) Logistic regression using SAS: Theory and application. USA: SAS Institute.

Arriagada, I. (2005) Dimensiones de la pobreza y polticas desde una perspectiva de género. Revista de la CEPAL (85), pp. 101-113.

Atkinson, A. B. (1987) On the measurement of poverty. Econometrica, 55(4), pp. 749-764. 
Bateman, I. J., Carson, R. T., Day, B., Hanemann, M., Hanley, N., Hett, T., Sugden, R. (2002) Economic valuation with stated preference techniques: A manual. Reino Unido: Edward Elgar Publishing.

Boltvinik, J. (2003) Tipología de los métodos de medición de la pobreza. Los métodos combinados. Comercio exterior 5 .

Castillo, D., Arzate, J. (2013) Crisis económica, pobreza y política social en México. Aportes 18(47), pp. 57-82.

CONEVAL. (2010) Metodología para la medición multidimensional de la pobreza en México 2009. México: Consejo Nacional de Evaluación de la Política de Desarrollo Social.

CONEVAL. (2011) Pobreza en México y en las entidades federativas. México: Consejo Nacional de Evaluación de la Política de Desarrollo Social.

CONEVAL. (2016) Informe de Evaluación de la política de desarrollo social 2018. México: Consejo Nacional de Evaluación de la Política de Desarrollo Social.

Córdova-Rivera, G. (2011) Comentarios y reseñas del libro: La Medición del Progreso y del Bienestar. Propuestas desde América Latina. Polis, Revista de la Universidad Bolivariana 29, pp. 465-470.

Cortés, F. (1997) Determinantes de la pobreza de los hogares. México, 1992. Revista Mexicana de Sociología, 2, 131-160.

Cortés, F., Hernández-Laos, E. (2004) Medición de la pobreza. DemoS, 15, pp. 7-9.

Damián, A. (2010) A dónde nos ha llevado el enfoque relativo de la pobreza de Peter Townsend. Mundo siglo XXI 19, 25-34.

Damián, A. (2015) Crisis global, económica, social y ambiental. Estudios demográficos y urbanos 30 (1), pp. 159-199.

Damián, A, Boltvinik, J. (2003) Evolución y características de la pobreza en México. Comercio Exterior 53 (6).

Destremau, B., Salama, P. (2002) La pobreza enredada en las turbulencias macroeconómicas en América Latina. Espiral 9 (25), pp. 178-227.

DOF. (2010) Lineamientos y criterios generales para la definición, identificación y medición de la pobreza. México: Diario Oficial de la Federación.

Fernández-Vega, C. (2013) México S.A. La jornada, 11 de abril de 2013, p. 4A.

Greene, W. (2003) Econometric Analysis. Ed. 5. USA: Prentice Hall.

Gujarati, D. (2004) Econometría. Ed. 5. México: McGraw-Hill. 
Hair, J., R., A. (1995) Multivariate data analysis. E.U.A: Macmillan.

Kennedy, P. (1992) A guide to econometrics. Oxford: Blackwell.

Mandeville, P. (2008) La razón de momios 2. Regresón Logística. Ciencia UANL $1(1)$.

Martínez, F., Quintana, L., R., V. (2015) Aálisis macroeconómico de los efectos de la liberalización financiera y comercial sobre el crecimiento económico de México, 1988-2011. Perfiles latinoamericanos 23 (45), pp. 79-104.

Melgar, N., M., R. (2011) Percepción individual de bienestar y desempeño macroeconómico. En M. Rojas (coordinador), La medición del progreso y del bienestar: propuestas desde América Latina, pp. 155-168.

Moscote, O., Rincón, W. (2012) El modelo lineal de probabilidad y el modelo logit, un ejemplo aplicado. Comunicaciones en Estadística 2(5).

Ocampo, J. A. (2005) Más allá de las reformas: dinámica estructural y vulnerabilidad macroeconómica. Colombia: CEPAL/Alfaomega .

Ponce, G. (2012) Vulnerabilidad social y riesgo de caer en pobreza en México. En F. Sales-Heredia [coordinador], Pobreza y factores de vulnerabilidad social en México pp. 15-54.

Pucutay-Vásquez, F. (2002) Los modelos Logit y Probit en la investigación social: el caso de la pobreza del Perú en el año 2001. Lima: Centro de Investigación y Desarrollo del Instituto Nacional de Estadística e Informática.

Rodríguez-Donate, C., Cáceres-Hernández, J. J. (2007) Modelos de elección discreta y especificaciones ordenadas: una reflexión metodológica. Estadística Española (166).

Sagarnaga-Villegas, L., Salas-González, J. M. (2011) Metodología para medir resultados de la iniciativa de apoyo integral para la superación de la pobreza y levantamiento basal: Informe final, Trabajo de Campo y Análisis de Línea Basa. México: Secretaria de Desarrollo Social.

Sen, A. (1992) Sobre conceptos y medidas de pobreza. Comercio Exterior (4).

Sen, A. (2001), Development as freedom. Great Britain: Oxford University Press.

Tezanos, J. F., Tezanos, S. (2004) Tendencias Sociales 1995-2003. Madrid: Fundación sistema.

Townsend, P. (1993) The International Analysis of Poverty. Harverster, London: Harvester Wheatsheaf. 